\title{
Equivalent Network for H-plane Rectangular Waveguide Circuit Based on Mode Theory and its Application for Analysis of Circuit Performance with Network Theory
}

\author{
Takaharu Hiraoka Kenichi Matsumoto Hsu, Jui-Pang \\ Faculty of Engineering, Kanagawa University, Yokohama Japan \\ hiraoka@cc.kanagawa-u.ac.jp
}

\begin{abstract}
Equivalent network of H-plane rectangular waveguide circuit consisting of straight waveguide and stepdiscontinuity is rigorously given by cascade connection of the corresponding equivalent network ,i.e, multi-transmission line and multiport ideal transformer. Thus obtained whole equivalent network of $H$-plane rectangular waveguide circuit in line is useful for systematic analysis of circuit performance and synthesis of desired characteristics.
\end{abstract}

\section{Introduction}

H-plane rectangular waveguide circuits as shown in Fig. 1 have been frequently used for realizing various function such as shown in Fig.3. So far these circuits have been treated by dominant mode equivalent network whose parameter is given by Waveguide Handbook, or recently by generalized $S$ matrix method. Latter method is effective and powerful for calculation of frequency characteristics, but is not appropriate for calculation of field distribution under operation. Instead, we propose to use rigorous mode corresponding equivalent network for straight waveguide and step discontinuity, which are already given by multi-transmission line and multiport ideal transformer, respectively ${ }^{[1]}$. Therefore, whole equivalent network for rectangular waveguide circuit in line is given by cascade connection of these equivalent network.

In the following it is explained how to apply the equivalent network with network theory for calculation of frequency characteristics, excited mode amplitude and 2$D$ field distribution under operation and extraction of useful parameters such as effective reactance for related modes. Through our network analysis, mode voltage $S$ matrix and mode power $S$ matrix are introduced which leads to the generalized $S$ matrix.

Finally, the above mentioned equivalent networks are applied to the analysis of practical structures.

\section{Description of Field by Planar Circuit Equations}

H-plane rectangular waveguide circuits can be understood as a parallel plate planar circuit with short circuited side wall, where the circuit has only $E_{z}$ and $\boldsymbol{H}_{t}\left(=H_{x}\right.$, $H_{y}$ ) components and can be described by the following planar circuit equations $(1)$, assuming sinusoidal $(\omega)$ and dominant mode $\left(\mathrm{TE}_{10}\right)$ excitation.

$$
\left\{\begin{array}{l}
\operatorname{grad} \dot{\boldsymbol{V}}=-j \omega \mu_{0} d \dot{\boldsymbol{J}} \\
\operatorname{div} \dot{\boldsymbol{J}}=-j \frac{\omega \varepsilon_{0}}{d} \dot{V}
\end{array}\right.
$$

where planar voltage $\dot{V}(x, y)$ and current density $\dot{\boldsymbol{J}}(x, y)$ are defined by electromagnetic field as in eqs.(2).

$$
\left\{\begin{array}{l}
\dot{V}(x, y)=-\dot{E}_{z}(x, y) \cdot d \\
\dot{\boldsymbol{J}}(x, y)=\dot{\boldsymbol{H}}_{t}(x, y) \times \boldsymbol{k}
\end{array}\right.
$$

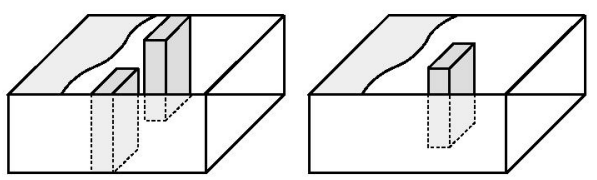

(a) Inductive window and inductive post

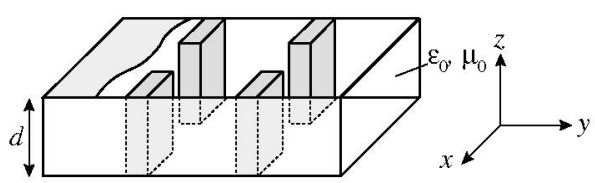

(b) Waveguide coupled cavity

Fig.1 Examples of H-plane rectangular waveguide discontinuity and circuit

\section{Equivalent Network for Waveguide Circuit}

H-plane rectangular waveguide circuit shown in Fig.1 or Fig. 3 can be treated by planar circuit equation (1) but here instead whole circuit is divided into two key elements, i.e. straight waveguide and step discontinuity as shown in Fig. 2 and 3. Equivalent networks for these two key elements have been derived rigorously based on mode analysis ${ }^{[1]}$.

\section{(1) Straight waveguide (Fig.2A)}

Equivalent network for $i$-th straight waveguide of width $W^{i}$ is given by mode corresponding multi-transmission line as shown in Fig.2A(b) where the mode voltage and current are related by the following transmission line equations.

$$
\begin{cases}\frac{d V_{p}^{i}\left(\ell^{i}\right)}{d \ell^{i}}=-j X_{p}^{i} I_{p}^{i}\left(\ell^{i}\right) & X_{p}^{i}=\frac{\omega \mu_{0} d}{W^{i}} \\ \frac{d I_{p}^{i}\left(\ell^{i}\right)}{d \ell^{i}}=-j B_{p}^{i} V_{p}^{i}\left(\ell^{i}\right) & B_{p}^{i}=\frac{\left(\beta_{p}^{i}\right)^{2} W^{i}}{\omega \mu_{0} d}\end{cases}
$$

where $p$-th mode function and mode propagation constants / characteristics impedance are defined by eqs.(4)

$$
\begin{aligned}
& S_{p}\left(s^{i}\right)=\sqrt{2} \sin \frac{p \pi s^{i}}{W^{i}} \quad\left(C_{p}\left(s^{i}\right)=\sqrt{2} \cos \frac{p \pi s^{i}}{W^{i}}\right) \\
& \beta_{p}^{i}=\sqrt{k_{0}^{2}-\left(\frac{p \pi}{W^{i}}\right)^{2}}, Z_{C_{p}}^{i}=\frac{\omega \mu_{0} d}{\beta_{p}^{i} W^{i}}, \quad \gamma_{p}^{i}=j \beta_{p}^{i}
\end{aligned}
$$

Vector notation for the equivalent network is shown in Fig.2(c) where $\boldsymbol{Z}_{C}^{i}, \boldsymbol{\gamma}^{i}, \boldsymbol{v}, \boldsymbol{i}^{i}$ are defined by matrix form.

$$
\left\{\begin{array}{l}
\boldsymbol{Z}_{C}^{i}=\operatorname{diag}\left(Z_{C 1}^{i}, Z_{C 2}^{i}, \cdots\right) \quad \gamma=\operatorname{diag}\left(\gamma_{1}^{i}, \gamma_{2}^{i}, \cdots\right) \\
\boldsymbol{v}^{i}\left(\ell^{i}\right)=\left(V_{1}^{i}(\ell), V_{2}^{i}(\ell), \cdots\right)^{t} \quad \boldsymbol{i}^{i}=\left(I_{1}^{i}(\ell), I_{2}^{i}(\ell), \cdots\right)^{t}
\end{array}\right.
$$

Through following analyses $j$-th port mode voltage/current column matrix at $i$-th waveguide are defined as

$$
v^{i, j}=\left(V_{1}^{i, j}, V_{2}^{i, j}, \cdots\right)^{t}, i^{i, j}=\left(I_{1}^{i, j}, I_{2}^{i, j}, \cdots\right)^{t}(j=1,2)(6)
$$

(2) Step discontinuity (Fig.2B)

Mode voltage and mode current at both sides of the step discontinuity shown in Fig. $2 \mathrm{~B}\left(a^{\prime}\right)$ are related by 
eqs.(7) based on mode orthogonality, which leads to the equivalent network shown in Fig. $2 \mathrm{~B}\left(b^{\prime}\right)$ by virtue of multiport ideal transformers.

$$
\begin{gathered}
I_{q}^{2}=\sum_{p=1}^{\infty} n_{q p}^{21} I_{p}^{1} \quad(q=1, \cdots, \infty) \quad V_{p}^{1}=\sum_{q=1}^{\infty} n_{q p}^{21} V_{q}^{2} \quad(p=1, \cdots, \infty) \\
\text { where } n_{q p}^{21}=\frac{1}{W^{1}} \int_{0}^{W_{2}} S_{q}\left(s^{2}\right) S_{p}\left(s^{1}\right) d s^{2}
\end{gathered}
$$

Hence, $n_{q p}^{21}$ means ideal transformer ratio between $q$ th mode in waveguide $\# 2$ and $p$-th mode in waveguide \#1. Equivalent network for step discontinuity becomes multiport ideal transformers shown in Fig.2( $\left.b^{\prime}\right)$ or Fig.2(c') in vector notation, where

$$
i^{2}=n^{21} i^{1}, \quad v^{1}=\left(n^{21}\right) v^{2}, \quad n=\left(n_{q p}^{21}\right)
$$

(3) Equivalent network for various waveguide circuits

Using the equivalent network for key elements, overall equivalent network of $\mathrm{H}$-plane rectangular waveguide circuits in Fig. 3 are given by cascade connection of the corresponding equivalent network in the same figure. In this way the field problem of a microwave circuit given by eq.(1) is transformed into a network problem.

\section{General analysis of circuit by network theory}

The field behavior of microwave circuit is fully described by thus obtained equivalent network. Hence, circuit performance such as frequency characteristics, amplitude of higher mode excited at discontinuity and time dependent 2-D field distribution can be calculated by the equivalent network with conventional network theory.

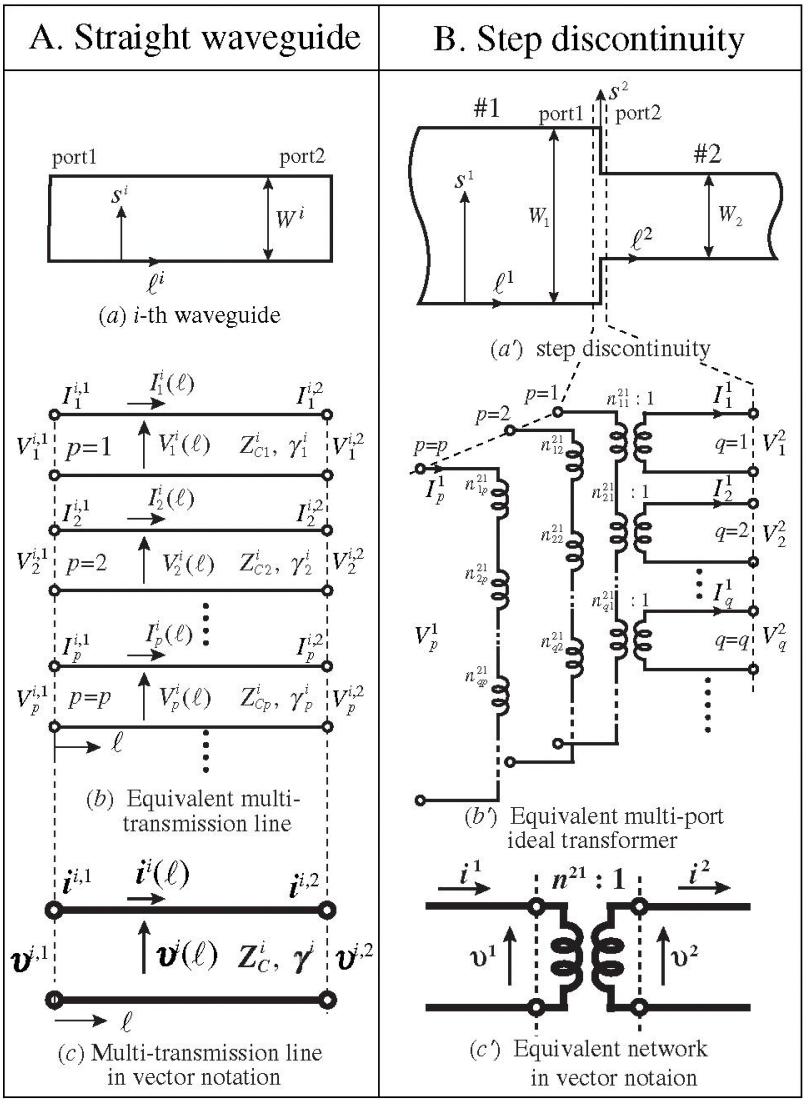

Fig.2 Equivalent network for waveguide(A) and step(B)

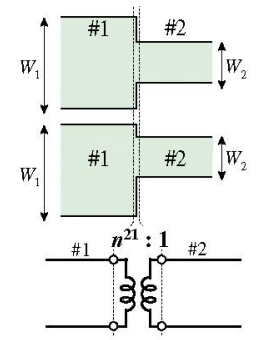

(a) Symmetric/Asymmetric step

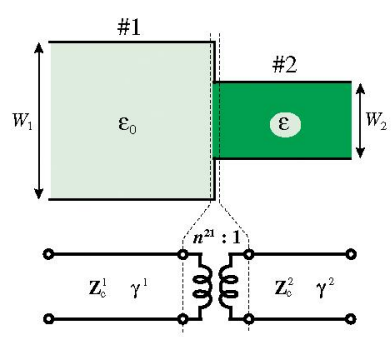

(b) Step discontinuity with different dielectric

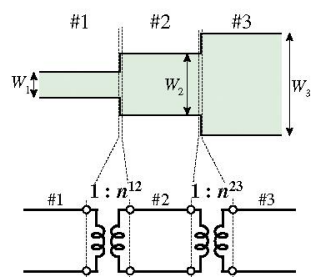

(e) Impedance transformer

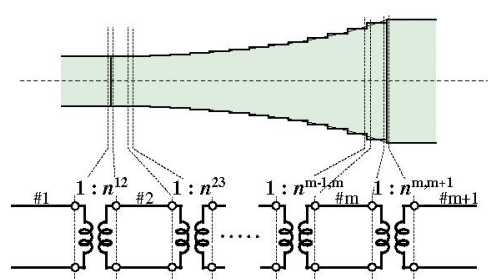

(f) Stair-case approximated taper

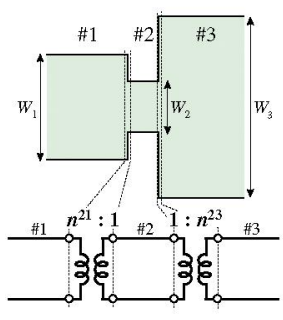

(c) Inductive window

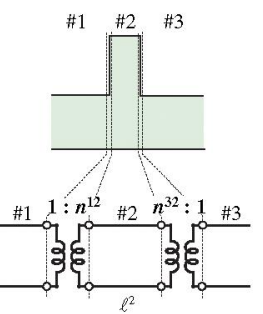

(g) H-plane stub

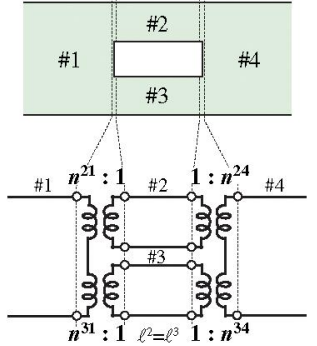

(d) Inductive rectangular post
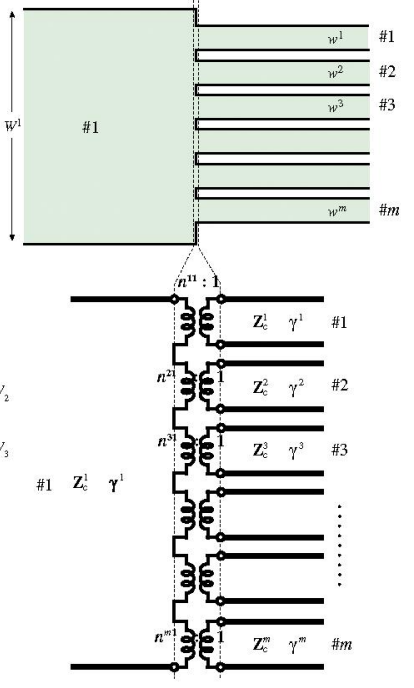

(k) m-output port power divider

Fig.3 Equivalent network for various $\mathrm{H}$-plane rectangular waveguide circuits 
In order to show how to use them, three examples such as inductive window / post and waveguide coupled cavity shown in Fig.1 are treated, whose whole equivalent networks are shown in Fig. 4( $n=3,4,5$ for each case which $n$ means number of waveguide related).

(1) External port and internal port for waveguide circuit

Waveguide circuits always have external waveguide for input/output and internal waveguide for realizing function. In Fig.4 \#1 and \#n waveguide are external waveguides, and \#2 to \#(n-1) are internal waveguides. We can define external port and internal port. For example $(1,2)^{*}$ and $(n, 1)$ are always external ports and the rest are internal ports in Fig.4. Then, we can define external and internal column matrix of mode voltage/current. " $(1,2)$ means port 2 of waveguide \#1.

\section{(2) External effective mode impedance matrix}

Mode voltage / current column matrix at external port defined by eqs. (14) are related by external effective mode impedance matrix $\boldsymbol{Z}_{e f f}^{e e}$, which can be obtained by application of network theory to the corresponding equivalent network.

$$
\boldsymbol{v}^{e}=\boldsymbol{Z}_{e f f}^{e e} \cdot \boldsymbol{i}^{e}
$$

(3) Mode voltage/power scattering matrix $\left(\boldsymbol{S}_{V}, \boldsymbol{S}_{P}\right)$

The incident/reflected mode voltage column matrix $A^{\text {ij, }}$, $B^{i, j}$ and the corresponding mode voltage/current column matrix at $j$-th port of $i$-th waveguide are related by following eqs.(11). ( $i=$ waveguide number, $j=$ port number)

$$
\boldsymbol{v}^{i, j}=\boldsymbol{A}^{i, j}+\boldsymbol{B}^{i, j} \quad \boldsymbol{i}^{i, j}=\left(\boldsymbol{A}^{i, j}-\boldsymbol{B}^{i, j}\right) \boldsymbol{Y}_{C}^{i}
$$

(a) Inductive window ( $\mathrm{n}=3$ )
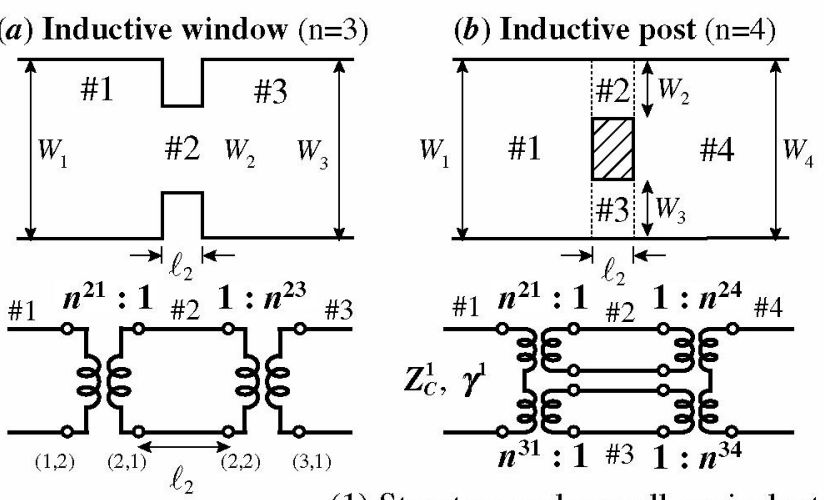

(1) Structure and overall equivalent networks for each case
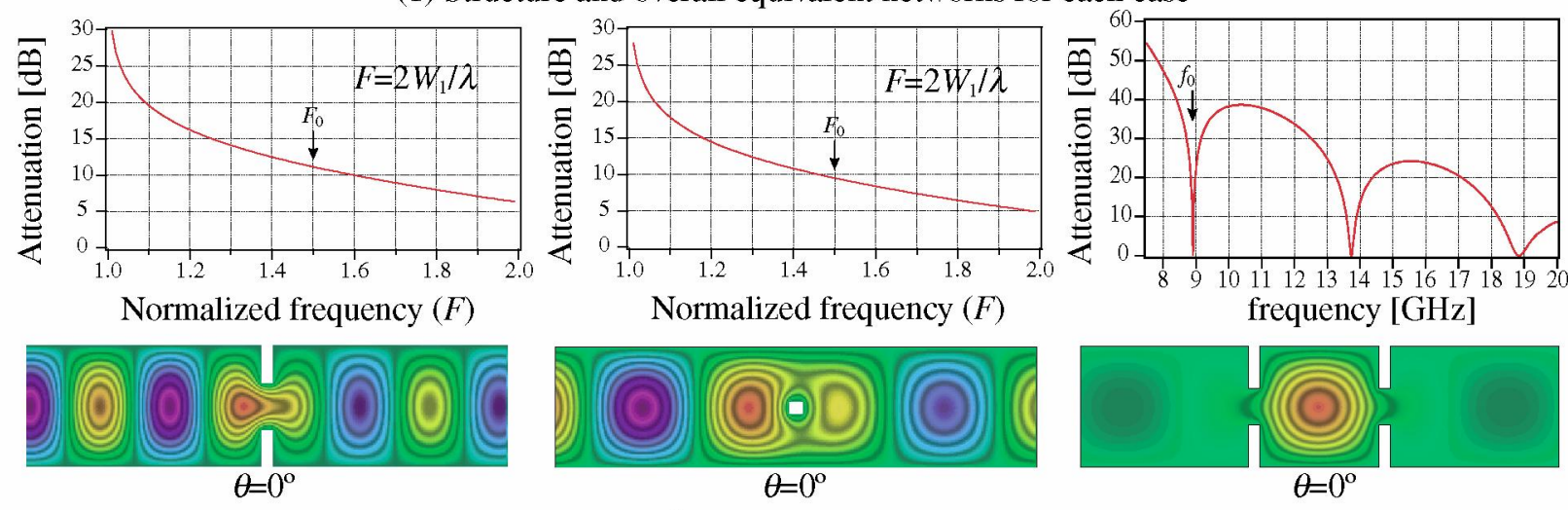

$t / W_{1}=0.1, W_{1}=W_{2}, W_{2} / W_{1}=0.4$

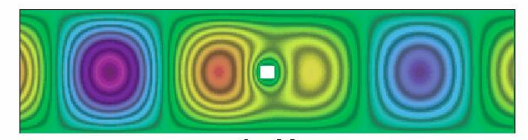

$\theta=0^{\circ}$

$t / W_{1}=0.1, W_{1}=W_{4}$
$W_{2} / W_{1}=W_{3} / W_{1}=0.4$

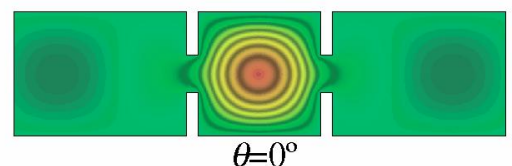

$t=2.0 \mathrm{~mm}, W_{2}=W_{4}=7.0 \mathrm{~mm}$

$W_{1}=W_{3}=W_{5}=23.1 \mathrm{~mm}$

(2) Dimension, frequency characteristics and $2 \mathrm{D}$ voltage distribution under operation at center frequency $\left(F_{0}\right.$ or $\left.f_{0}\right)$

Fig.4 Equivalent network, frequency characteristics and voltage distribution for H-plane waveguide circuit 
$\boldsymbol{n}=\left[\begin{array}{cc}n^{21} & 0 \\ 0 & n^{23}\end{array}\right] \quad Z_{t}^{2}=\left[\begin{array}{ll}Z_{C}^{2} \operatorname{coth} \gamma^{2} \ell_{2} & Z_{C}^{2} \operatorname{csch} \gamma^{2} \ell_{2} \\ Z_{C}^{2} \operatorname{csch} \gamma^{2} \ell_{2} & Z_{C}^{2} \operatorname{coth} \gamma^{2} \ell_{2}\end{array}\right]$

$Z_{t}^{2}$ is transmission line mode impedance matrix of waveguide $\# 2$.

$Z_{\text {eff }}^{e e}$ of inductive post shown in Fig.4(b) are derived in the same manner and given by the same form as eq.(15)

(2) Waveguide coupled cavity (Fig.4(c))

Waveguide coupled cavity and its equivalent network are shown in Fig.4(c). We define two two-port external effective mode impedance matrices at waveguide \#2 and \#4 ${ }^{2} Z_{t},{ }^{4} Z_{t}$, which are given by similar equation as eq.(15). Then external effective mode impedance matrix of the overall network is given by eq.(17)

$$
\boldsymbol{Z}_{e f f}^{e e}=\left[\boldsymbol{Z}^{e e}-\boldsymbol{Z}^{e i}\left(\boldsymbol{Z}^{e i}+\boldsymbol{Z}_{t}^{3}\right)^{-1} \boldsymbol{Z}^{i e}\right]
$$

where $Z^{e e}, Z^{e i}, Z^{i e}, Z^{i i}$ are defined by the matrix eq.(18).

$$
\begin{gathered}
{\left[\begin{array}{c}
v^{(1,2)} \\
v^{(5,1)} \\
v^{(3,1)} \\
v^{(3,2)}
\end{array}\right]=\left[\begin{array}{cccc}
{ }^{2} \boldsymbol{Z}_{t}^{(1,1)} & 0 & { }^{2} \boldsymbol{Z}_{t}^{(1,2)} & 0 \\
0 & { }^{4} \boldsymbol{Z}_{t}^{(2,2)} & 0 & { }^{4} \boldsymbol{Z}_{t}^{(2,1)} \\
{ }^{2} \boldsymbol{Z}_{t}^{(2,1)} & 0 & { }^{2} \boldsymbol{Z}_{\boldsymbol{t}}^{(2,2)} & 0 \\
0 & { }^{4} \boldsymbol{Z}_{\boldsymbol{t}}^{(1,2)} & 0 & { }^{4} \boldsymbol{Z}_{\boldsymbol{t}}^{(1,1)}
\end{array}\right]\left[\begin{array}{l}
\overrightarrow{\boldsymbol{i}}^{(1,2)} \\
\overline{\boldsymbol{i}}^{(5,1)} \\
\overline{\boldsymbol{i}}^{(3,1)} \\
\overrightarrow{\boldsymbol{i}}^{(3,2)}
\end{array}\right]} \\
\therefore\left[\begin{array}{c}
\boldsymbol{v}^{e} \\
\boldsymbol{v}^{i}
\end{array}\right]=\left[\begin{array}{ll}
\boldsymbol{Z}^{e e} & \boldsymbol{Z}^{e i} \\
\boldsymbol{Z}^{i e} & \boldsymbol{Z}^{i i}
\end{array}\right]\left[\begin{array}{l}
\boldsymbol{i}^{e} \\
\boldsymbol{i}^{i}
\end{array}\right]
\end{gathered}
$$

Frequency response described by $S_{P}$ for three structures shown in Fig. 4 are given by substituting the corresponding $Z_{e f f}^{e e}$ into eq.(13). The detail is explained at IV, (3).

\section{Calculation of 2-D Field distribution}

When waveguide circuit is excited by incident mode voltage $A^{1,2}, A^{n, 1}$ at external port of \#1 and \#n waveguide , reflected mode voltage $B^{1,2}, B^{n, 1}$ at external port can be calculated by mode voltage scattering matrix (13), and then mode voltage/ current at external port are given by eq.(11). Internal port mode voltage/current can be calculated by substituting external port mode voltage/current into the equivalent network. Calculated results of excited mode amplitude at each port for waveguide coupled cavity shown in Fig. 4(c) with dominant mode incidence of unit amplitude at port 1 at resonant frequency are shown in Fig.5. It is interesting that incident mode in the cavity is almost dominant mode but reflected waves include higher modes as shown by table in Fig. 5 . Also 2-D voltage and current distribution in waveguide circuit can be calculated by product of mode voltage/ current distribution along transmission line with mode function. 2-D voltage distribution for unit amplitude incident of dominant mode at port 1 are calculated and shown in Fig.4 for three cases(50 modes are considered).

\section{Conclusion}

Equivalent network for $\mathrm{H}$-plane rectangular waveguide circuits are rigorously given by cascade connection of equivalent network of key element, i.e. waveguide and step discontinuity. Thus obtained whole equivalent network for waveguide circuit will be useful for systematic analysis and synthesis of H-plane rectangular waveguide circuit, because it fully describes character of field and network in the waveguide circuit. These equivalent networks are applied to H-plane rectangular waveguide discontinuity/circuits with success.

\section{Reference}

[1] Hsu, J.P., T.Hiraoka, H.Honma "Equivalent network for rectangular-waveguide H-plane step discontinuity Multi-transmission and multi-port ideal transformer-" 2000 IEEE MTT-S Digest WEIF-3 pp1069-1072

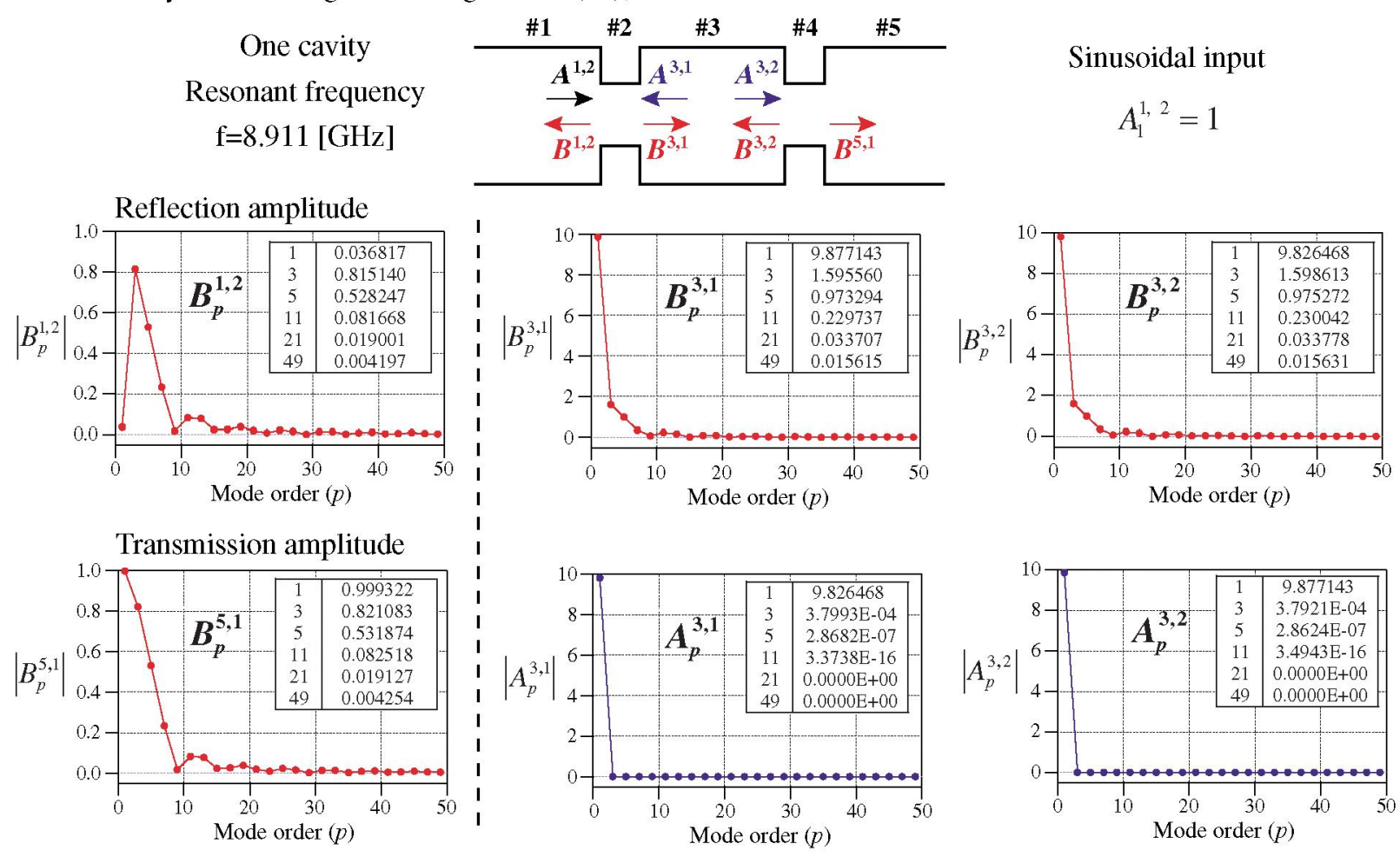

Fig.5 Incident and reflected amplitude of dominant and higher order modes at each port - waveguide coupled cavity (dimension are given in Fig.4) - 